\title{
A NEW INTERACTIVE METHOD FOR CORONARY ARTERIES SEGMENTATION BASED ON TUBULAR ANISOTROPY
}

\author{
Fethallah Benmansour, Laurent D. Cohen \\ CEREMADE, UMR CNRS 7534, Université Paris Dauphine, \\ Place du Maréchal De Lattre De Tassigny, 75775 PARIS CEDEX 16 - FRANCE \\ \{benmansour, cohen\}@ceremade.dauphine.fr
}

\begin{abstract}
In this paper we present a new interactive method for tubular structure extraction. The main application and motivation for this work is vessel tracking in 3D medical images. The basic tools are minimal paths solved using the fast marching algorithm. This leads to interactive tools for the physician by clicking on a small number of points in order to obtain a minimal path between two points or a set of paths in the case of a tree structure. Our method is based on a variant of the minimal path method that models the vessel as a centerline and surface by adding one dimension for the local radius around the centerline. The crucial step of our method is the definition of the local metrics to minimize (based on the local orientation using a Riemannian Metric). This approach is made available for the physician using an Object Oriented Language (OOL) interface. We show results on two CT cardiac images for coronary arteries segmentation.
\end{abstract}

Index Terms - Image segmentation, Image enhancement, Medical diagnosis

\section{INTRODUCTION}

Coronary artery disease (CD) is the leading cause of death in the United States. In symptomatic patients, diagnosis of the presence and severity of coronary artery disease is critical for determining appropriate clinical management. Indirect evaluation of coronary stenosis, such as through stress testing, has limited diagnostic ability as compared with direct conventional coronary angiography. Conventional coronary angiography reveals the extent, location, and severity of coronary obstructive lesions, which are potent predictors of outcome, and identifies high-risk patients who may benefit from revascularization. Thus, invasive coronary angiography, despite the associated risks, remains the standard for the diagnosis of obstructive coronary artery disease. Multidetector computed tomographic (MDCT) angiography has been proposed as a noninvasive test to determine the presence of $\mathrm{CD}$. Although MDCT angiographic data acquisition is straightforward, effective visualization and communication of the complex multifocal manifestations of CD remain a major challenge. For accurate image interpretation, precise, fast and semiautomatic image processing tools are mandatory.
This need has attracted the attention of the computer vision community yielding to multidisciplinary collaborations. Moreover, segmentation of tubular structures like blood vessels or coronary arteries is a very active research domain since the early 90's. Indeed, various methods such as vascular image enhancement methods [1-3], or others were proposed, see [4] for a complete survey.

Siddiqi and Vasilevskiy proposed in [5] a vessel segmentation method based on flux maximizing flows. On the same research line, Bouix et al proposed in [6] a method for automatic centerline extraction once the boundaries of the vessel are found. Nonetheless, their approach suffers from huge time consuming due to the used level set formulation and to the used iterative algorithm. Moreover, the inward flux they maximize is not concave at all with respect to the evolving shape and may be stuck on local maxima yielding to bad result. Cohen and Kimmel introduced in [7] the minimal path method that captures the global minimum curve between two points given by the user. This leads to the global minimum of an active contour energy. Using the Fast Marching method [8], the minimal path problem can be solved efficiently. The main drawback of the minimal path method when it is applied to vessel segmentation is that it provides only a trajectory (or a set of trajectories), and does not give any information about the vessel boundary and local width. In [9], Deschamps and Cohen used the image itself as a propagation potential while they applied the Fast Marching method to an excellent contrast air-filled colon on CT scanner and considered the propagating front as an estimate of the vessel boundary. However, classical segmentation problems do not provide such an excellent contrast yielding the propagating front to flow over the boundaries of longer and thinner objects. Again, in [9], authors proposed to extract the centerline using the estimated vessel boundaries.

Our method is based on a variant of the classical, purely spatial, minimal path technique by incorporating an extra non-spatial dimension into the search space. This approach was first proposed by Li et al [10]. Each point of the 4D path (after adding the extra dimension for the 3D image) consists of three spatial coordinates plus a fourth coordinate which de- 
scribes the vessel thickness at that corresponding point. Thus each 4D point represents a sphere in 3D space, and the vessel is obtained by taking the envelope of these spheres as we move along the 4D curve. The main drawback of their method is that they considered only isotropic media that does not take into account the orientation of the vessels. Our first contribution is to take into account the vessel orientation by defining a suited anisotropic metric that makes the propagation faster along the center lines and for the adequate radius. The second contribution is to build an anisotropic metric based on the Optimally Oriented Flux (OOF) descriptor. The OOF was first presented by Law et al in [11] for vessel enhancement. Its main advantage is that the disturbance introduced by the closely located nearby structures is avoided. But they did not exploit the orientations given by the OOF. We propose to do so by using the OOF's scalar functions and its orientation. That makes the propagation faster along the vessel's center line and for exact associated scale. This means that the path location, orientation and scale have to be coherent with the local geometry of the image extracted by the OOF.

In section 2, we give some background on minimal path method and Anisotropic Fast Marching. In section 3 the Optimally Oriented Flux descriptor is presented as well as the metric construction. In section 4 , we will show segmented coronary arteries using our method. Finally, conclusions and perspectives follow in section 5 .

\section{BACKGROUND ON MINIMAL PATH METHOD}

A minimal path, first introduced in the isotropic $(\mathcal{P}$ does not depend on the orientation of the path) case [7], is a pathway minimizing the energy functional :

$$
E(\gamma)=\int_{\gamma} \mathcal{P}\left(\gamma(s), \gamma^{\prime}(s)\right) \mathrm{d} s
$$

where, $\mathcal{P}\left(\gamma, \gamma^{\prime}\right)=\sqrt{\gamma^{\prime T} \mathcal{M}(\gamma) \gamma^{\prime}}$ describes an infinitesimal distance along a pathway $\gamma$ relative to a metric tensor $\mathcal{M}$ (symmetric definite positive). $\mathcal{M}$ is defined from some features extraction in the image. A curve connecting $\mathbf{p}_{1}$ to $\mathbf{p}_{2}$ that globally minimizes the above energy (1) is a minimal path between $\mathbf{p}_{1}$ and $\mathbf{p}_{2}$, noted $\mathcal{C}_{\mathbf{p}_{1}, \mathbf{p}_{2}}$. The solution of this minimization problem is obtained through the computation of the minimal action map $\mathcal{U}: \Omega \rightarrow \mathbb{R}^{+}$associated to $\mathbf{p}_{1}$ on the domain $\Omega$ which can be a 2D, 3D or $4 \mathrm{D}$ domain (in the case of $3 \mathrm{D}$ tubular structures, $\Omega$ is a $4 \mathrm{D}$ domain). The minimal action is the minimal energy integrated along a path between $\mathbf{p}_{1}$ and any point $\mathbf{x}$ of the domain $\Omega$ :

$$
\forall \mathbf{x} \in \Omega, \mathcal{U}_{1}(\mathbf{x})=\min _{\gamma \in \mathcal{A}_{\mathbf{p}_{1}, \mathbf{x}}}\left\{\int_{\gamma} \mathcal{P}\left(\gamma(s), \gamma^{\prime}(s)\right) \mathrm{d} s\right\},
$$

where $\mathcal{A}_{\mathbf{p}_{1}, \mathbf{x}}$ is the set of smooth paths linking $\mathbf{x}$ to $\mathbf{p}_{1}$. The values of $\mathcal{U}_{1}$ may be regarded as the arrival times of a front propagating from the source $\mathbf{p}_{1}$ with oriented velocity related to the metric tensor $\mathcal{M}^{-1} . \mathcal{U}_{1}$ satisfies the Eikonal equation

$$
\left\|\nabla \mathcal{U}_{1}(\mathbf{x})\right\|_{\mathcal{M}^{-1}(\mathbf{x})}=1 \text { for } \mathbf{x} \in \Omega \text {, and } \mathcal{U}_{1}\left(\mathbf{p}_{1}\right)=0,
$$

where $\|\mathbf{v}\|_{M}=\sqrt{\mathbf{v}^{T} M \mathbf{v}}$. The flow lines of $\mathcal{U}_{1}$ satisfy the Euler-Lagrange equation of functional (1). Thus, the minimal path $\mathcal{C}_{\mathbf{p}_{1}, \mathbf{p}_{2}}$ can be retrieved with a simple gradient descent on $\mathcal{U}_{1}$ from $\mathbf{p}_{2}$ to $\mathbf{p}_{1}$ (see Fig. 1). Proof of (3) can be found in [8].

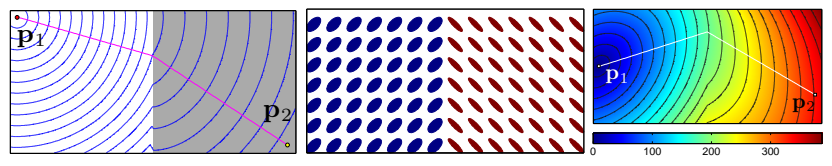

Fig. 1. Minimal path examples on an isotropic case on the left image. On the middle, visualization by small ellipses of eigenvalues of a metric constant on each half side of the image, and the minimal action map associated to the source point $\mathbf{p}_{1}$ with the minimal path $\mathcal{C}_{\mathbf{p}_{1}, \mathbf{p}_{2}}$ on the right.

On figure 1, we show some examples of the minimal path method on an isotropic case and an anisotropic one. On the first image of figure 1 the metric is isotropic and the potential in the grey region is twice as low as the white one. Isolevel sets of the minimal action map associated to the source point $\mathbf{p}_{1}$ and the minimal path $\mathcal{C}_{\mathbf{p}_{1}, \mathbf{p}_{2}}$ are displayed. The second image represents a metric $\mathcal{M}$. We took two constant metrics in each half side of the image with different orientations. On the last image, the minimal action map $\mathcal{U}_{1}$ associated to the metric $\mathcal{M}$ and to the source point $\mathbf{p}_{1}$ is shown. The minimal path $\mathcal{C}_{\mathbf{p}_{1}, \mathbf{p}_{2}}$ is found through a simple gradient descent. The anisotropic Eikonal equation is solved using an adapted version of the Fast Marching algorithm. Descriptions and details on the isotropic and anisotropic Fast Marching can be found in $[7,8]$.

\section{OPTIMALLY ORIENTED FLUX : AN ANISOTROPY DESCRIPTOR}

We are interested in the construction of a metric that extracts from the image the geometric information leading to reconstruction of vessels. This means that we wish to find an estimate for the local orientation and scale and a criterion on the local geometry to distinguish the presence of vessels from the background.

At the position $\mathbf{x}$ on an image $I$, the amount of the image gradient projected along the axis $\mathbf{v}$ flowing out from a $3 \mathrm{D}$ sphere $S_{r}$ of radius $r$ is measured as in [11],

$$
f(\mathbf{x}, \mathbf{v} ; r)=\int_{\partial S_{r}}((\nabla(G * I)(\mathbf{x}+\mathbf{h}) \cdot \mathbf{v}) \mathbf{v}) \cdot \frac{\mathbf{h}}{|\mathbf{h}|} \mathrm{d} a,
$$

where $G$ is a Gaussian function with a scale factor equal to the size of the image voxel, $\mathbf{h}$ is the position vector along $\partial S_{r}$ and $\mathrm{d} a$ is the infinitesimal area on $\partial S_{r}$. To detect vessels having higher intensity than the background region, one would be interested in finding the vessel direction which minimizes $f(\mathbf{x}, \mathbf{v} ; r)$,

$$
\arg \min _{\mathbf{v}} f(\mathbf{x}, \mathbf{v} ; r) \text {. }
$$


Using the divergence theorem, it can be shown that $f(\mathbf{x}, \mathbf{v} ; r)$ can be calculated using a simple convolution,

$$
f(\mathbf{x}, \mathbf{v} ; r)=\mathbf{v}^{T}\left\{\left(\partial_{i, j} G\right) * I * \mathbb{1}_{S_{r}}\right\} \mathbf{v},
$$

where $\left(\partial_{i, j} G\right)$ is the Hessian matrix of function $G$ and $\mathbb{1}_{S_{r}}$ is the indicator function inside the sphere $S_{r}$. By differentiating the above equation with respect to $\mathbf{v}$, the solution of equation (5) is in turn acquired as solving a generalized eigenvalue decomposition problem. Solving the aforementioned generalized eigen decomposition problem gives three eigenvalues, $\lambda_{1}(\cdot) \leq \lambda_{2}(\cdot) \leq \lambda_{3}(\cdot)$ and three eigenvectors $\mathbf{v}_{i}(\cdot)$, i.e. $\lambda_{i}(\mathbf{x} ; r)=f\left(\mathbf{x}, \mathbf{v}_{i}(\mathbf{x} ; r) ; r\right)$ for $i=1,2,3$. The two eigenvectors associated to the first eigenvalues $\left(\lambda_{1}, \lambda_{2}\right)$ represent directions that are orthogonal to the vessel. $\mathbf{v}_{3}$ represents the direction along the vessel. To handle the vessels having various radii, a multi-scale approach should be employed along with the OOF method. In [11], Law and Chung have proposed to normalize the OOF's eigenvalues by the sphere surface area $\left(4 \pi r^{2}\right)$ when the OOF method is incorporated in a multi-scale approach for 3D image volumes.

$\mathrm{Li}$ and Yezzi [10] proposed a new variant of the classical, purely spatial, minimal path technique by incorporating an extra non-spatial dimension into the search space. The crucial step of this method is to build an adequate metric that drives the propagation. Li and Yezzi [10] proposed different isotropic potentials. The main drawback, as they mention, is that these potentials are very parameter dependent and they do not exploit the vessel orientation. Our main contribution is to improve $\mathrm{Li}$ and Yezzi method by adding to it an anisotropic formulation, and the anisotropic metric is constructed by extension of the OOF descriptor presented by Law et al [11].

The 4D minimal path is found by minimizing the following energy:

$$
\int_{\gamma}\left\{\sqrt{\gamma^{\prime}(s)^{T} \mathcal{M}(\gamma(s)) \gamma^{\prime}(s)}\right\} \mathrm{d} s,
$$

where $\mathcal{M}$ is the $4 \mathrm{D}$ anisotropic metric we want to construct. It not natural to consider anisotropy on the fourth dimension, i.e the radii dimension. Thus one can decompose by block the metric $\mathcal{M}$ as follows :

$$
\mathcal{M}(\mathbf{x}, r)=\left(\begin{array}{cc}
\tilde{\mathcal{M}}(\mathbf{x}, r) & 0 \\
0 & \mathcal{P}_{\text {radii }}(\mathbf{x}, r)
\end{array}\right)
$$

where $\tilde{\mathcal{M}}(\mathbf{x}, r)$ is a $3 \times 3$ symmetric definite positive matrix giving the spatial anisotropy and $\mathcal{P}_{\text {radii }}(\mathbf{x}, r)$ is the radii potential (also strictly positive).

Since the result given by the anisotropic minimal path method is very dependent on the metric, results inherit advantages and drawbacks of the constructed metric, thus we should be very carful with its construction. First, let us fix conditions on the desired metric. The spatial metric $\tilde{\mathcal{M}}$ has to be well oriented along the vessel centerline. And the radii potential $\mathcal{P}_{\text {radii }}$ has to be small for the adequate scale for any point of the image. $\mathcal{P}_{\text {radii }}$ corresponds to the inverse speed for the radii dimension. Since $\tilde{\mathcal{M}}$ is symmetric definite positive, we can decompose it as follows:

$$
\tilde{\mathcal{M}}(.)=\sum_{i=1}^{3} m_{i}(.) \mathbf{u}_{i}(.) \mathbf{u}_{i}(.)^{T},
$$

where $0<m_{1} \leq m_{2} \leq m_{3}$ are the eigenvalues and $\mathbf{u}_{i}$ are the associated eigenvectors. The velocity of the propagating front along direction $\mathbf{u}_{i}$ is equal to $1 / \sqrt{m_{i}}$. We used the OOF descriptor to construct the metric as follows:

$$
\left\{\begin{aligned}
\tilde{\mathcal{M}}(.) & =\sum_{i=1}^{3} \exp \left(\alpha \frac{\sum_{j \neq i} \lambda_{j}(.)}{2}\right) \mathbf{v}_{i}(.) \mathbf{v}_{i}(.)^{T}, \\
\mathcal{P}_{\text {radii }}(.) & =\beta \exp \left(\alpha \frac{\sum_{i=1}^{3} \lambda_{i}(.)}{3}\right) .
\end{aligned}\right.
$$

The constants $\alpha$ and $\beta$ are controlled by two intuitive parameters, which are the maximal spatial anisotropy ratio and radii speed ratio respectivelly.

\section{RESULTS}

Our method is minimally interactive. First, the user has to decide if the desired vessels are darker or brighter than the background. So, we can consider different criteria on signs of the eigenvalues. Then the scale range $\left[r_{\min }, r_{\max }\right]$, which corresponds to the range of radii of the vessel one wants to extract, is given by the user. Finally few points are required as source points or end points of the Fast Marching algorithm. We used the metric described in the previous section to find the minimal anisotropic path (as described in section 2) between two or more selected points (see figure 2). For any selected point, the associated radius is equal to the minimal radius $r_{\min }$ given by the user. One figure 2, left anterior descending (LAD) arteries and right coronary arteries (RCA) are segmented.

One can see that the obtained radii on the principal coronary branches are larger than those of the secondary. Thus, our approach is robust to scale changing and bifurcations. Nevertheless, our current implementation requires huge memory allocations due to the $4 \mathrm{D}$ and anisotropic aspects. To overcome this issue, we added a pre-processing interactive tool to select a subvolume containing the desired vessels (see figure 2). Moreover, we are working on a new implementation of the tubular anisotropy approach to make the memory allocation dynamic and hence to benefit from the front propagation aspect of the fast marching algorithm. Besides the reduction of the computation time (which has been actually achieved), we will save on memory allocation and will have a new version of our algorithm that extract the whole coronary arteries using a regular PC.

\section{CONCLUSIONS}

In this paper we have proposed a new method for 3D tubular structure extraction. Our method exploit the orientations 


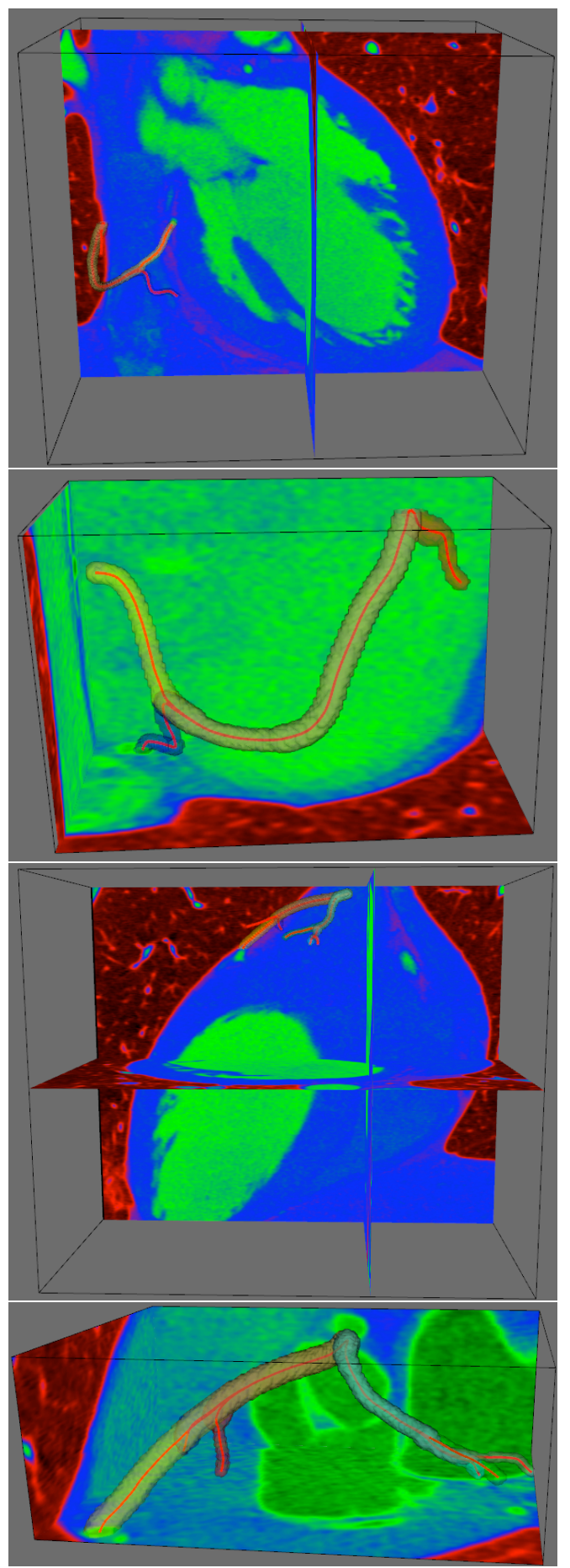

Fig. 2. First two images : RCA segmentation using the tubular anisotropy approach shown on the whole image and on the selected sub-volume. Second two images : LAD segmentation shown on the whole image and on the selected subvolume. Only few points are required (the extremities of the paths). The tubular anisotropy method provides the centerline as well as vessels boundaries. of the vessels by using the optimally oriented flux to construct a multi-resolution anisotropic metric that describes the vessels orientation and scales. Combining this metric with anisotropic minimal path technique, we are able to find a complete description of the tubular structure, i.e the center line and the boundary. To summarize, our method is minimally interactive, robust to scale variations and to bifurcations. We developed a user friendly interface for coronary arteries segmentation and we are working on its medical validation.

\section{Acknowledgements}

We would like to thank Professor Anthony J. Yezzi, Max WaiKong Law and Professor Philippe Charles Douek for fruitful discussions. Also, Eduardo Davila for his precious help for the implementation of the interface. This work was partially supported by ANR grant SURF -NT05-2_45825. and the France-Israel project?

\section{REFERENCES}

[1] Y. Sato, S. Nakajima, N. Shiraga, H. Atsumi1, S. Yoshida, T. Koller, G. Gerig, and R. Kikinis, "Threedimensional multi-scale line filter for segmentation and visualization of curvilinear structures in medical images," MedIA, vol. 2, no. 2, pp. 143-168, 1998.

[2] K. Krissian, "Flux-based anisotropic diffusion applied to enhancement of 3D angiogram," TMI, vol. 21, no. 11, pp. 1440-1442, 2002.

[3] Ro F. Frangi, Wiro J. Niessen, Koen L. Vincken, and Max A. Viergever, "Multiscale vessel enhancement filtering," 1998, vol. 1496, pp. 130-137, Springer-Verlag.

[4] Cemil Kirbas and Francis K. H. Quek, "A review of vessel extraction techniques and algorithms," ACM Computing Surveys, vol. 36, pp. 81-121, 2004.

[5] K. Siddiqi and A. Vasilevskiy, "3d flux maximizing flows," in EMMCVPR02, 2002, p. $636 \mathrm{ff}$.

[6] Sylvain Bouix, Kaleem Siddiqi, and Allen Tannenbaum, "Flux driven automatic centerline extraction," Medical Image Analysis, vol. 9, no. 3, pp. 209-221, 2005.

[7] L. D. Cohen and R. Kimmel, "Global minimum for active contour models: a minimal path approach," Int J Comput Vision, vol. 24, pp. 57-78, 1997.

[8] J. A. Sethian and A. Vladimirsky, "Fast methods for the eikonal and related hamilton- jacobi equations on unstructured meshes," Proceedings of the National Academy of Sciences, vol. 97, no. 11, pp. 5699-5703, May 2000.

[9] T. Deschamps and L.D. Cohen, "Fast extraction of minimal paths in 3D images and applications to virtual endoscopy," MIA, vol. 5, no. 4, Dec. 2001.

[10] H. Li and A. Yezzi, "Vessels as 4D curves: Global minimal 4D paths to extract 3D tubular surfaces and centerlines," IEEE TMI, vol. 26, no. 9, pp. 1213-1223, 2007.

[11] Max W. K. Law and Albert C. S. Chung, "Three dimensional curvilinear structure detection using optimally oriented flux," ECCV, vol. 4, pp. 368-382, 2008. 